\title{
LETTER OPEN \\ Mutations of MSH5 in nonobstructive azoospermia (NOA) and rescued via in vivo gene editing
}

Signal Transduction and Targeted Therapy (2022)7:1

\section{Dear Editor,}

Male infertility is a multifactorial heterogeneous pathological condition affecting $\sim 7 \%$ of men. Nonobstructive azoospermia (NOA) is one of the most severe male reproductive diseases and occurs in $\sim 1 \%$ of men of reproductive age. ${ }^{1}$ However, its etiology remains elusive.

Meiosis is a germ cell-specific cell division process. MSH5 (MutS homolog 5) is a member of the MutS family and is known to be involved in DNA mismatch repair. MSH5 acts with the MutS homolog partner MSH4 to form the MutS heterodimer. It has been demonstrated that the loss of Msh4 or Msh5 results in the earlier loss of prophase I progression, with the almost complete failure of homologous synapsis and cell death prior to pachynema. ${ }^{2,3}$ In a previous study, we identify a homozygous missense mutation (c.1459G > T, p.D487Y) of MSH5 gene in a pedigree of primary ovarian insufficiency (POI). ${ }^{4}$ In this study, homozygous frameshift truncation and compound heterozygous missense mutations of MSH5 were identified in three NOA pedigrees.

Three NOA patients (P8944, P7602, and P7824) came from three Chinese families without a history of fertility problems. Known causal factors for male infertility, including cryptorchidism, hypogonadism, cancer, drinking, and smoking, were excluded. Physical examination showed that the development of the penis, epididymis, prostate, scrotum, and vas deferens was normal. The testis size and the FSH level of the three probands were relatively normal. No defect of the 46, XY karyotype or microdeletions on the $Y$ chromosome were detected in these patients (Supplementary Table 1). The results of semen analysis and hematoxylin and eosin (H\&E) staining showed that normal sperm were absent in all three probands (Supplementary Table 2 and Supplementary Fig. 1a). TUNEL-positive apoptotic cells were detected in seminiferous tubules (Supplementary Fig. 1b).

To explore the genetic cause of infertility, three probands were chosen for whole-exome sequencing. Frameshift deletion of the MSH5 gene (c.678_681del, p.Y227Vfs*21) was identified in proband P8944 (Supplementary Table 3). Sanger sequencing (sequencing of blood samples) showed that proband P8944 carried a homozygous frameshift truncation mutation of $\mathrm{MSH} 5$ (c.678_681del). His parents, brother, and sister all carried a heterozygous deletion (Fig. 1a and Supplementary Fig. 2a). The deletion of TTAC causes a frameshift at codon 227 that results in a premature stop codon at codon 247 (Supplementary Fig. 2b). The truncated protein lost 587 amino acids at C-terminal, including the ATP-binding domain. The ATP-binding domain has been demonstrated to be essential for MSH5 function. ${ }^{2}$ We consider this deletion to be equivalent to a null allele, and the inactivation of MSH5 causes defects in germ cell development in the mouse model. ${ }^{2}$ Therefore, we conclude that the four-nucleotide deletion is the pathological cause of NOA in patient P8944.

Compound heterozygous mutations of the MSH5 gene were identified in patients P7824 and P7602 (Supplementary Tables 4 and 5). Both of them carried a common point mutation c.1459G $>T$
; https://doi.org/10.1038/s41392-021-00710-4

(p.D487Y), which we previously identified in the POI pedigree. ${ }^{4}$ In addition to this common mutation, patient P7602 carried another missense mutation, c.830C > T (p.P277L), and patient P7824 carried point mutation at 1914 (c.1914C>A, p.C638*) which causes early termination of translation (Fig. 1a and Supplementary Fig. 2c). The positions of all these mutations are conserved among mammalians (Fig. 1b). Furthermore, the MSH5 c.1459G >T mutation that has been identified in patients with $\mathrm{POI}$ was detected in the fathers of both patients, and female mice carrying the homozygous mutation (p.D486Y) are infertile with atrophic ovaries. ${ }^{4}$

To test the function of this mutation, we examined germ cell development in male mice carrying homozygous Msh5 mutation. We found that the homozygous Msh5 mutant (p.D486Y) male mice were infertile and the size of testes was dramatically reduced, and a large number of TUNEL-positive apoptotic cells were noted in the seminiferous tubules of $M s h 5^{D 486 Y / D 486 Y}$ mice (Fig. 1c). It is worth noting that the difference of testes size between mice and patients could be due to the different functions of MSH5 in human and mouse. The number of germ cells was significantly reduced in Msh5 $5^{\text {D486Y/D486Y }}$ mice from P14 (Supplementary Fig. 3). The pathological change observed in Msh5 $5^{D 486 Y / D 486 Y}$ mice was similar to that in patients P7602 and P7824, indicating that this mutation is a possible etiological cause of NOA.

To explore the causes of the defect of germ cell development in $M s h 5^{D 486 Y / D 486 Y}$ mice, the expression of meiosis-associated genes was examined by immunofluorescence. We found that the number of germ cells at the zygotene stage was significantly increased, whereas that at the diplotene and diakinesis stages was significantly decreased in Msh5 $5^{D 486 Y / D 486 Y}$ mice. And the proportion of unsynapsed homologous chromosomes was increased in Msh5 $5^{\text {D486Y/D486Y }}$ mice $(50.42 \pm$ $1.15 \%)$ at the diplotene stage compared to that in control mice $(14.14 \pm 2.92 \%)$ (Supplementary Fig. 4). These results indicate that the formation of the synaptonemal complex (SC) is not affected in Msh5 mutant germ cells. However, this mutation affects the stability of the $\mathrm{SCs}$, which leads to early desynapsis of homologous chromosomes.

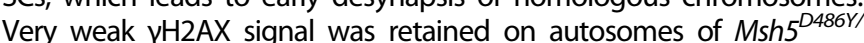
${ }^{D 486 Y}$ germ cells at the pachytene and diplotene stages, and a small number of RAD51and DMC1 foci were retained in Msh5 mutant spermatocytes at diplotene (Supplementary Fig. 5), indicating that the DSB is not completely repaired. The point mutation of $\mathrm{MSH} 5$ does not affect the interaction between the MSH4 and MSH5 proteins and the foci of MSH4 and MLH1 was virtually absent in Msh5 mutant germ cells (Fig. 1d and Supplementary Fig. 6). p.D487Y located in the DNAbinding domain and the original residue is highly conserved among species from yeast to human. We assume that the mutation could affect the combining capacity between MSH4-MSH5 and DNA. These results indicate that the homologous recombination does not occur in Msh5 mutant germ cells which is consistent with previous studies.

CRISPR/Cas system is the most powerful gene-editing technology which has been widely used in the treatment of different kinds of disease models. To rescue the defect of germ cell development in 
a

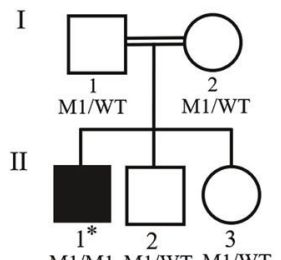

M1/M1 M1/WT M1/WT

M1:c.678_681del (p.Tyr227Valfs*21)

NOA

* Used for WES
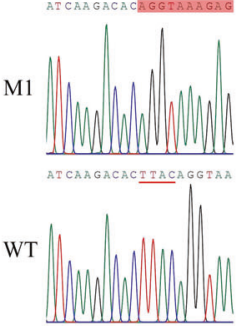

\section{P7602 family}

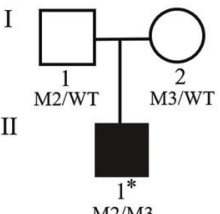

M2:c. 1459G $>$ T(p.D487Y)

M3:c. 830C $>$ T (p.P277L)
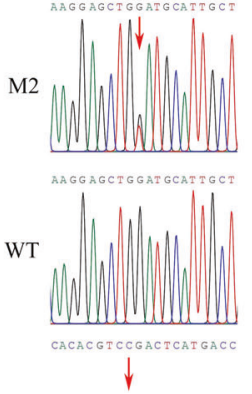

M3

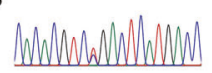

WT

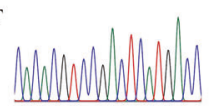

P7824 family

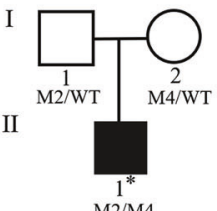

M2:c.1459G $>$ T(p.D487Y) M4:c.1914C $>$ A (p.C638*)
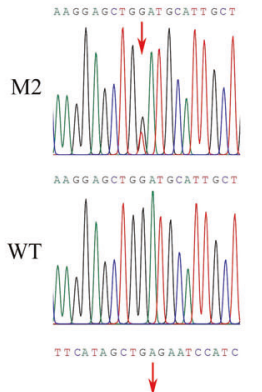

M4

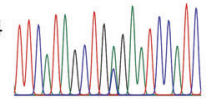

WT

b

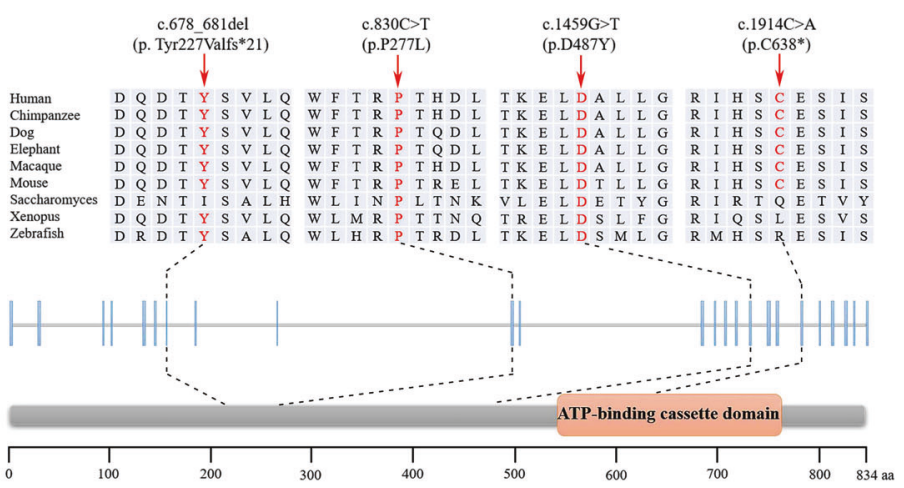

f

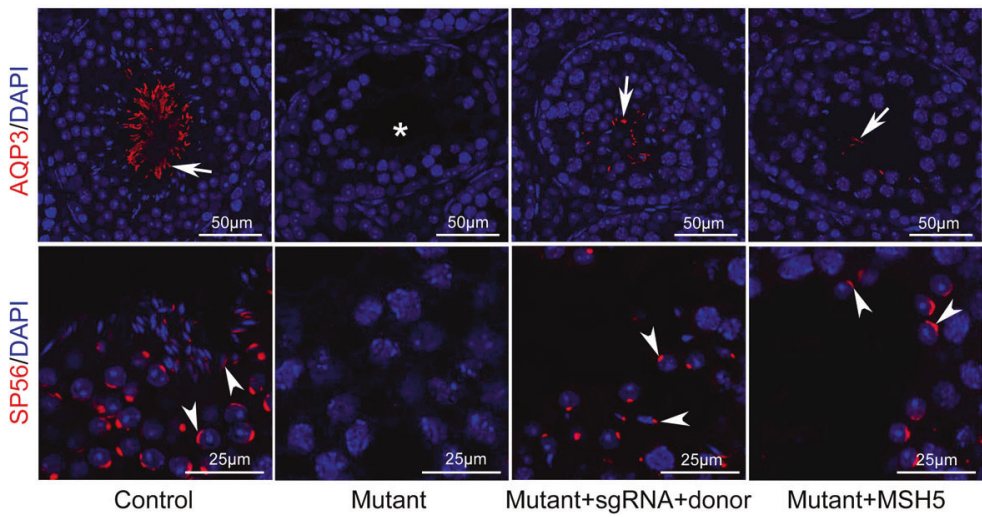

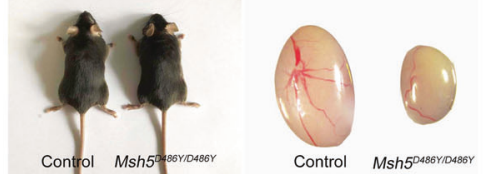
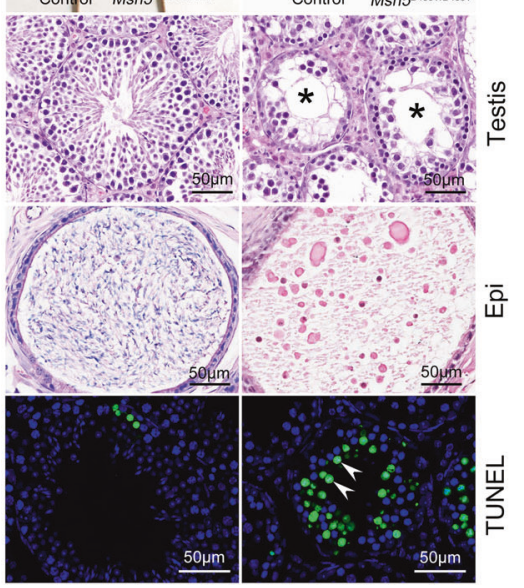

Control

Msh5 D486YID486Y

d

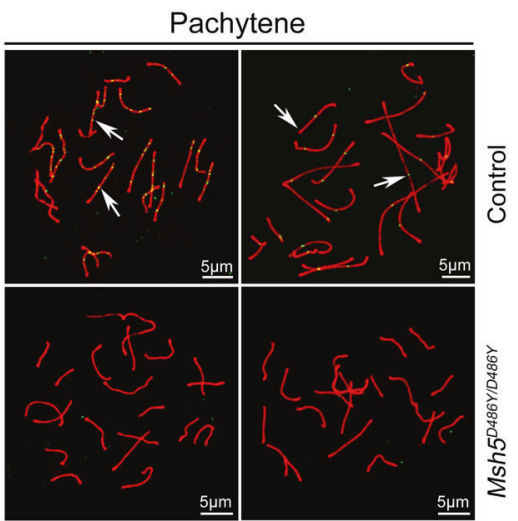

MSH4/SYCP3

MLH1/SYCP3

e

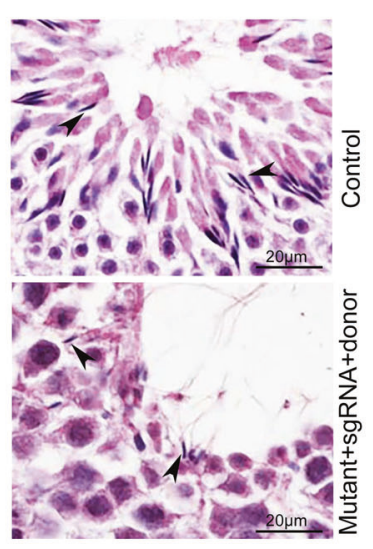

Msh5 $5^{\text {D486/D486Y }}$ mice, we established an in vivo electroporation system as described previously ${ }^{5}$ (Supplementary Figs. 7a and 8a). The mixture of guide RNA, Cas9-expressing vector, and single-stranded DNA template (donor) or wild-type Msh5-expressing vector was injected into the seminiferous tubules. The testes were electroporated after injection to promote the efficiency of transfection. Interestingly, a small number of mature sperm with head and tail were noted in the seminiferous tubules of Msh5 mutant testes after electroporation 5 weeks later (Fig. 1e, f). However, no mature sperm were obtained from epididymis cauda. This is probably due to the relatively low 
Fig. 1 Mutations of MSH5 in NOA pedigrees and gene editing. a The mutations (M1-M4) in MSH5 were identified in three probands with NOA (P8944, P7602, and P7824). WES was performed in the NOA patients (indicated by asterisks), and the genotypes of other family members were examined by Sanger sequencing. M1 is a frameshift with four nucleotides deletion. M2-M4 are missense point mutations. Red box indicates the shifted sequences, and the deleted nucleotides (TTAC) are indicated with red underline. Red arrows indicate the positions of point mutations. Double horizontal lines represent consanguineous marriages. WT wild-type, $\mathrm{M}$ mutant type. $\mathbf{b}$ The location and conservation of four mutations in MSH5 (M1-M4) protein. c The defect of germ cell development in male Msh5 $5^{D 486 Y / D 486 Y}$ mice. Msh5 $5^{D 486 Y / D 486 Y}$ male mice were viable at birth and no obvious developmental defects were observed in adults. The size of testes from adult Msh5 $5^{D 486 Y / D 486 Y}$ mice was significantly reduced compared to that of control littermates. Control testis sections showed normal cell populations within the seminiferous epithelium, whereas germ cell loss no round and elongating spermatids were observed in Msh5 $5^{D 486 Y / D 486}$ mice (asterisks). A large number of mature sperm were observed in the epididymis cauda of control mice. Only cell debris was observed in the epididymis cauda of Msh $5^{D 486 Y /}$ ${ }_{D 486 Y}$ mice. Very few TUNEL-positive germ cells were observed in control testes and the number of apoptotic cells was significantly increased in Msh5 $5^{D 86 Y / D 486 Y}$ testes (arrowheads). Scale bars: $50 \mu \mathrm{m}$. Experiments were repeated $\geq 3$ times. d Immunostaining of MSH4 and MLH1 was performed in chromosome spreads of control and Msh5 $5^{D 486 Y / D 486 Y}$ germ cells at P30. Synaptonemal complex was labeled with SYCP3 (red). MSH4 and MLH1 foci were observed in control germ cells at the pachytene stage. No MSH4 and MLH1 foci were observed in Msh5 mutant germ cells at the pachytene stage. Scale bars: $50 \mu \mathrm{m}$. Experiments were repeated $\geq 3$ times. e H\&E staining of control and rescued (with guide RNA, Cas9-expressing vector, and donor DNA) testes. The germ cells at different developmental stages were observed in control testes. A small number of mature sperm were noted in the seminiferous tubules of rescued testes (black arrows). Scale bars: $20 \mu \mathrm{m}$. Experiments were repeated $\geq 3$ times. f Immunofluorescence analysis of AQP3 and SP56 in control and rescued mutant mice. Mature sperm were also observed in the Msh5 mutant testes after electroporation with the Msh5-expressing vector. The sperm tail was labeled with AQP3 (white arrows). The acrosome of sperm head was labeled with SP56 (white arrowheads). Scale bars: $50 \mu \mathrm{m}, 25 \mu \mathrm{m}$. Experiments were repeated $\geq 3$ times

efficiency of gene correction with this system. The delivery system is needed to be optimized to increase the transfecting efficiency (the experiment is repeated more than three times, and the number of animals is more than ten). Approximately $9 \%$ of $\mathrm{G}>\mathrm{T}$ mutation was corrected in Msh5 mutant testes electroporated with the guide RNA, Cas9-expressing vector, and donor DNA fragment, and no off-target effects were detected by DNA sequencing (Supplementary Fig. 8 and Supplementary Table 6). These results indicate that the mutation in the germ cells of Msh5 $5^{D 486 Y / D 486 Y}$ mice could be corrected by in vivo gene editing.

Taken together, our findings based on both human subjects and mouse models strongly suggest that the mutation of MSH5 is a potential etiological cause of NOA. Most importantly, we have corrected the Msh5 mutation in the mouse model via in vivo gene editing. This approach will be potentially applied in the clinic treatment of male reproductive diseases.

\section{DATA AVAILABILITY}

The online version of this article contains supplementary material, which is available to authorized users.

\section{ACKNOWLEDGEMENTS}

This work was supported by the National Key R\&D Program of China (2018YFA0107702, 2018YFC1004200); Strategic Priority Research Program of the Chinese Academy of Sciences (XDB19000000); The National Science Fund for Distinguished Young Scholars (81525011); Strategic Collaborative Research Program of the Ferring Institute of Reproductive Medicine, Ferring Pharmaceuticals and Chinese Academy of Sciences (FIRMC180307); The National Natural Science Foundation of China (31970785 and 31671496).

\section{ADDITIONAL INFORMATION}

Supplementary information The online version contains supplementary material available at https://doi.org/10.1038/s41392-021-00710-4.

Competing interests: The authors declare no competing interests.

Min Chen ${ }^{1}$, Chencheng $\mathrm{Yao}^{2}$, Yingying Qin ${ }^{3}$, Xiuhong Cui ${ }^{4}$, Peng $\mathrm{Li}^{2}$, Zhiyong $\mathrm{Ji}^{2}$, Limei Lin ${ }^{4,5,6}$, Haowei Wu ${ }^{4,5,6}$, Zhi Zhou ${ }^{\prime}$ Yaoting Gui ${ }^{1 凶}$, Zheng $\mathrm{Li}^{2 \bowtie}$ and Fei Gao (iD) $4,5,6$ 两

${ }^{1}$ Guangdong and Shenzhen Key Laboratory of Male Reproductive Medicine and Genetics, Institute of Urology, Peking University Shenzhen Hospital, Shenzhen Peking University-The Hong Kong

University of Science and Technology Medical Center, Shenzhen, Guangdong Province, P. R. China; ${ }^{2}$ Department of Andrology, the
Center for Men's Health, Urologic Medical Center, Shanghai Key Laboratory of Reproductive Medicine, Shanghai General Hospital, Shanghai Jiao Tong University School of Medicine, Shanghai, P. R. China; ${ }^{3}$ Center for Reproductive Medicine of Shandong University, National Research Center for Assisted Reproductive Technology and Reproductive Genetics, The Key Laboratory for Reproductive Endocrinology of Ministry of Education, Jinan, P. R. China; ${ }^{4}$ State Key

Laboratory of Stem cell and Reproductive Biology, Institute of Zoology, Chinese Academy of Sciences, Beijing, P. R. China, ${ }^{5}$ University of Chinese Academy of Sciences, Beijing, P. R. China;

${ }^{6}$ Institute for Stem Cell and Regeneration, Chinese Academy of Sciences, Beijing, China and 7 School of Life Science and Technology, ShanghaiTech University, Shanghai, P. R. China

These authors contributed equally: Min Chen, Chencheng Yao, Yingying Qin. Correspondence: Yaoting Gui (guiyaoting2007@aliyun.com) or Zheng Li (lizhengboshi@163.com) or Fei Gao (gaof@ioz.ac.cn)

\section{REFERENCES}

1. Cerván-Martín, M., Castilla, J. A., Palomino-Morales, R. J. \& Carmona, F. D. Genetic landscape of nonobstructive azoospermia and new perspectives for the clinic. J. Clin. Med. 9, 300 (2020)..

2. Edelmann, W. et al. Mammalian MutS homologue 5 is required for chromosome pairing in meiosis. Nat. Genet. 21, 123-127 (1999).

3. Kneitz, B. et al. MutS homolog 4 localization to meiotic chromosomes is required for chromosome pairing during meiosis in male and female mice. Genes Dev. 14, 1085-1097 (2000).

4. Guo, T. et al. Mutations in MSH5 in primary ovarian insufficiency. Hum. Mol. Genet 26, 1452-1457 (2017).

5. Michaelis, M., Sobczak, A. \& Weitzel, J. M. In vivo microinjection and electroporation of mouse testis. J. Vis. Exp. 51802 (2014).

Open Access This article is licensed under a Creative Commons Attribution 4.0 International License, which permits use, sharing, adaptation, distribution and reproduction in any medium or format, as long as you give appropriate credit to the original author(s) and the source, provide a link to the Creative Commons license, and indicate if changes were made. The images or other third party material in this article are included in the article's Creative Commons license, unless indicated otherwise in a credit line to the material. If material is not included in the article's Creative Commons license and your intended use is not permitted by statutory regulation or exceeds the permitted use, you will need to obtain permission directly from the copyright holder. To view a copy of this license, visit http://creativecommons. org/licenses/by/4.0/.

(c) The Author(s) 2021 